\title{
HerRamientas PARA El CONTROL de LA disLiPEMIA en el paciente con DM2: dieta, ejercicio y fármacos
}

Daniel Escribano PARdo

Medicina Familiar y Comunitaria. Centro de Salud Oliver (Zaragoza).

\begin{abstract}
Resumen
La DM2 (diabetes mellitus tipo 2) guarda una estrecha relación en su aparición y pronóstico con el estilo de vida. A esta situación se suma la posible coexistencia con alteraciones a nivel del perfil lipídico en el paciente con DM2. El deterioro de los patrones dietéticos tradicionales, el sedentarismo y, como consecuencia, la obesidad crecientes en nuestra sociedad moderna han contribuido de un modo determinante al dramático y progresivo aumento de la prevalencia de DM2 así como alteraciones en el perfil lipídico que modifican el riesgo cardiovascular en general, y de estos pacientes en particular. Hacemos un repaso de las recomendaciones más recientes en relación a las medidas higiénico-dietéticas así como a las posibles medidas farmacológicas a implementar para controlar la dislipemia en el paciente con DM2.
\end{abstract}

Palabras clave: DM2, dislipemia, dieta, actividad física, fármacos hipolipemiantes, riesgo cardiovascular.

\section{Dieta}

Los patrones dietéticos que se han evaluado más ampliamente son la dieta DASH (Dietary Approaches to Stop Hypertension), baja en sal y con alta ingesta de frutas, vegetales, granos integrales, lácteos bajos en grasas y proteínas magras, y la dieta mediterránea. La diferencia más relevante es el énfasis de la segunda en el uso de aceite de oliva virgen extra. Un ensayo clínico de Prevención Primaria con dieta mediterránea ${ }^{1}$ demostró que los participantes aleatorizados a esta dieta, complementada con aceite de oliva virgen extra o frutos secos, tuvieron una incidencia un $30 \%$ menor de ECV (Eventos Cardiovasculares) en comparación con aquellos que estaban en el grupo de dieta baja en grasas. No hay grandes novedades en las recomendaciones para la intervención no farmacológica en el manejo de los pacientes con dislipemia en la actual guía ${ }^{2}$ respecto a la guía previa ${ }^{3}$. A modo de resumen podríamos recoger los siguientes aspectos:

- Se debe disminuir la ingesta calórica y aumentar el gasto energético en aquellas personas con exceso de peso o adiposidad abdominal. La reducción de 5-10\% de peso (que sería en principio un objetivo a fijar con el paciente) mejoraría las alteraciones lipídicas y afecta favorablemente a los otros FRCV (Factores de Riesgo Cardiovascular), como diabetes, hipertensión arterial, estado proinflamatorio...
- Se debe evitar cualquier consumo de grasas trans. Según la Fundación Española del Corazón, las fuentes de los ácidos grasos trans son dos: natural y artificial o industrial. La fuentie natural radica en los animales rumiantes, como la vaca, la oveja o la cabra. Aproximadamente un $5 \%$ del consumo total de ácidos grasos trans proviene del consumo de grasa de rumiantes (a través del consumo de productos como mantequilla, crema, nata, leche entera, carne grasa, etc.).

Los ácidos grasos trans industriales se generan al hidrogenar parcialmente los aceites vegetales. Los alimentos horneados como las galletas, la bollería industrial, los alimentos precocinados y los fritos como patatas fritas, maíz y otros aperitivos, junto con los helados, cremas y batidos son los alimentos que contienen grasas trans en mayor cantidad.

- El consumo de grasas saturadas debe ser $<10 \%$ del total ingesta calórica y debe reducirse aún más ( $<7 \%$ de energía) en presencia de hipercolesterolemia. Se debe aumentar el consumo de ácidos grasos monoinsaturados, así como los polinsaturados. La ingesta de colesterol en la dieta debe ser < 300 mg/día. En comparación con dietas altas en grasa, estas dietas ricas en hidratos de carbono reducen el colesterol total y el c-LDL en un 10-20 \%, 
pero pueden aumentar los triglicéridos si son altas en azúcares. También resultan útiles en el control del peso, dado que la disminución de la ingesta total de grasas conduce a la reducción de la ingesta de calorías. Durante mucho tiempo se ha recomendado la dieta baja en grasas para la prevención de la ECV, pero a pesar de las grandes expectativas que existían con este modelo de dieta, un estudio aleatorizado en más de 48.000 mujeres posmenopáusicas demostró que no reducía el riesgo de ECV, incluyendo ictus y enfermedad coronaria 4 .

- La ingesta de hidratos de carbono, cuyo efecto es neutro sobre el c-LDL, debe oscilar entre un $45 \%$ y un $55 \%$ del total de la ingesta de energía, ya que tanto los porcentajes más altos como los más bajos de hidratos de carbono se asocian con un aumento de la mortalidad. El consumo excesivo provoca efectos adversos en los TG (triglicéridos) plasmáticos y en los niveles de c-HDL.

- La fibra dietética, particularmente la de tipo soluble presente en legumbres, frutas, verduras y granos de cereales integrales (por ejemplo, avena y cebada), tiene un efecto hipocolesterolémico.

- La ingesta de azúcar añadida no debe exceder el 10 \% de la energía total. Los refrescos deben ser drásticamente limitados en aquellos individuos con niveles elevados de TG o adiposidad visceral.

- Un consumo moderado de alcohol, < 10 g/día (1 unidad) para hombres y mujeres, es aceptable para aquellos que toman bebidas alcohólicas si los niveles de TG no son elevados y no hay otra contraindicación formal para su consumo.

- No hay que olvidar, dentro de las medidas higiénico-dietéticas, que el abandono del hábito tabáquico tiene claros beneficios con respecto al riesgo general cardiovascular y, específicamente, en los niveles de c-HDL.

En relación a lo anteriormente expuesto recomendamos la actualización publicada por la Sociedad Española de Arteriosclerosis en cuanto al estilo de vida en la prevención cardiovascular ${ }^{5}$.

Otras indicaciones respecto a otros componentes de la dieta y suplementos son:

1. La ingesta media diaria de fitosteroles, presentes en aceites vegetales, verduras, frutas frescas, nueces, granos y legumbres, es de 500 mg en países mediterráneos. El consumo diario de suplementos (2 g) puede reducir los niveles del c-LDL un 7-10 \%, pero no se han realizado estudios sobre el efecto en la prevención de ECV. Estos suplementos pueden considerarse en:

- Individuos con c-LDL elevado y RCV intermedio o bajo, en los que no está indicado el uso de fármacos.

- Como complemento de los fármacos en pacientes con RCV alto y muy alto.

- En adultos y niños mayores de nueve años con hipercolesterolemia familiar como complemento a los fármacos.

2. La levadura de arroz rojo (LAR) contiene monacolina K, metabólicamente similar a la lovastatina. En el único estudio ${ }^{6}$ en pacientes con ECV, realizado en China, se redujeron los eventos recurrentes en un $45 \%$. Con LAR se alcanzan descensos del c-LDL, hasta un $20 \%$, por cada 5-10 mg de monacolina K. Puede considerarse en personas con niveles elevados en c-LDL en las que las recomendaciones actuales no aconsejan el uso de terapias con estatinas.

3. El consumo de pescado (al menos dos veces por semana) y de alimentos vegetales ricos en ácidos grasos omega-3 se ha asociado con un menor riesgo de muerte por ECV. La dosis de ácidos grasos omega-3 de cadena larga (2-3 g/día) reducen los niveles de triglicéridos (TG) alrededor del $30 \%$ y también la lipemia posprandial, pero unas dosis más altas pueden aumentar los niveles del c-LDL. En este sentido se debe hacer referencia a los resultados del estudio REDUCE-IT?'

\section{Actividad física}

La actividad física ${ }^{8}$ no solo tiene un efecto positivo en las personas con dislipemia, sino que también puede ayudar a mejorar el perfil de lípidos. El tiempo, el volumen y la intensidad del ejercicio tienen un efecto sobre los cambios inducidos por el ejercicio en los lípidos. El c-HDL es el más sensible al ejercicio. Para reducir más los niveles de c-LDL y TG, es necesario aumentar la intensidad del ejercicio aeróbico.

La actividad física regular y mantenida protege frente a la aparición de las ECV con una relación dosis-respuesta9 ${ }^{9}$. Sin embargo, desde el punto de vista cardiovascular y para la población general, se recomiendan actividades aeróbicas que sean moderadas y regulares. La actividad física moderada requiere un esfuerzo moderado que acelera de forma perceptible el ritmo cardíaco. 
Por lo tanto, se debe realizar ejercicio físico regular de moderada intensidad durante al menos $30 \mathrm{~min} /$ día, incluso si no se tiene sobrepeso. Mejora el perfil lipídico, especialmente reduce los TG y eleva el c-HDL (por ejemplo, caminar rápido $25-30 \mathrm{~km} / \mathrm{semana}$ puede aumentar los niveles de c-HDL en 3,16 mg/dl). Los pacientes adultos con diabetes deben realizar por lo menos 150 minutos semanales de ejercicio aeróbico de moderada intensidad (50 \% a 70 \% de la frecuencia cardíaca máxima calculada) distribuidos en la semana sin que haya más de dos días sin ejercicio entre ellos.

En ausencia de contraindicaciones, los pacientes con diabetes tipo 2 deben ser alentados a desarrollar entrenamiento contra resistencia por lo menos dos veces por semana ${ }^{10-11}$.

En un análisis post hoc del estudio ADVANCE (seguimiento cinco años) se correlacionó el nivel de ejercicio físico realizado en la semana previa a la aleatorización con la tasa de eventos cardiovasculares en 11.140 pacientes con diabetes tipo 2. Se observó que los sujetos que realizaron ejercicio físico moderado o vigoroso durante al menos 15 minutos por semana tuvieron una incidencia más baja de eventos cardiovasculares mayores (HR, cociente de riesgo o Hazard Ratio: 0,78; IC, Intervalo Confianza: $95 \%, 0,69-0,88 ; p<0,001$ ), menos complicaciones microvasculares (HR: 0,85; IC: 95 \%, 0,76-0,96; $p=$ 0,010) y menor mortalidad (HR: 0,83; IC: $95 \%$, 0,73$0,94 ; p=0,0044)$ que los pacientes que realizaron ejercicio físico leve o ninguna actividad física ${ }^{12}$. HUNT fue un estudio prospectivo observacional que incluyó a 56.170 personas mayores de 20 años seguidas durante una media de 12 años. El objetivo fue investigar el efecto de la actividad física desarrollada por los participantes durante su tiempo libre sobre la mortalidad cardiovascular y evaluar si la actividad física podría mitigar los efectos adversos de la diabetes. La diabetes se asoció con una mortalidad cardiovascular casi tres veces mayor en los pacientes físicamente inactivos. En cambio, el riesgo de muerte entre los pacientes con diabetes con actividad física de moderada a intensa fue similar al de sujetos sedentarios sin diabetes ${ }^{13}$.

\section{Fármacos hipolipemiantes en personas con diabetes}

Siguiendo la última guía de la ESC/EAS (Sociedad Europea de Cardiología/Sociedad Europea de Ateroesclerosis) ${ }^{2}$ sobre las recomendaciones del manejo de la dislipemia, a la hora de hablar de las personas con diabetes, hay distintos grados de recomendación de las diferentes medidas:

- Se recomienda o está indicado en pacientes con DM2 con riesgo muy alto una reducción de LDL-C de $\geq 50 \%$ con respecto al valor inicial y un objetivo de $c-L D L<55 \mathrm{mg} / \mathrm{dl}$ ). En pacientes con DM2 de alto riesgo, se recomienda una reducción de LDL-C de $\geq 50 \%$ desde el valor inicial y un objetivo de $c-L D L-C<70 \mathrm{mg} / \mathrm{dl}$. Se recomiendan las estatinas en pacientes con DM1 (diabetes mellitus 1) que tienen un riesgo alto o muy alto. Si los objetivos no se logran con la dosis máxima tolerada de estatina (clase de recomendación I y nivel de evidencia A), se recomienda la combinación con ezetimiba (clase de recomendación I y nivel de evidencia B). Para la prevención secundaria, si los pacientes con riesgo muy alto no logran su meta con una dosis máxima tolerada de estatina y ezetimiba, se plantea una combinación con un iPCSK9 (inhibidores de proproteína convertasa subtilisina/kexina tipo 9). Para pacientes con HF (hipercolesterolemia familiar) de muy alto riesgo (es decir, con ASCVD, riesgo de enfermedad cardiovascular aterosclerótica, o con otro factor de riesgo importante) que no logran sus objetivos con el máximo de la dosis tolerada de estatina y ezetimiba, se recomienda una combinación con un iPCSK9.

- Debería ser considerada la intensificación de la terapia con estatinas antes de la introducción de la terapia combinada. Si no se alcanza el objetivo, se debe considerar la combinación de estatinas con ezetimiba.

- No se recomienda la terapia con estatinas en pacientes premenopáusicas con DM que estén considerando quedarse embarazadas o no estén usando un método anticonceptivo adecuado.

Destaca la importancia de considerar las características específicas de la dislipemia en personas con DM y síndrome metabólico, afirmando que la dislipemia aterogénica ${ }^{14}$ es uno de los principales factores de riesgo de ECV en personas con DM2 y en personas con obesidad abdominal y resistencia a la insulina o con intolerancia a la glucosa. Los pacientes con diabetes presentan una dislipemia particularmente aterogénica ${ }^{15}$. Asocian un aumento de la concentración de apolipoproteína B debido a un incremento del número de las partículas contenidas en ella. La agresividad de esta dislipemia 
puede no ser evidente cuando uno analiza el perfil lipídico en la práctica clínica, ya que el LDL colesterol puede permanecer dentro del rango normal, siendo el colesterol no HDL el que puede evidenciar más claramente el riesgo. Aproximadamente un $50 \%$ de los pacientes con diabetes tipo 2 presentan en el perfil lipídico una disminución de HDL colesterol asociada a un aumento de los triglicéridos. El aumento de triglicéridos es característicamente basal y posprandial. Se indica que se considere el uso de estatinas solas o combinadas con otros hipolipemiantes para alcanzar los objetivos determinados. Además, se puede considerar el tratamiento con estatinas tanto en pacientes con DM1 como con DM2 con edades $\leq 30$ años, con evidencia de daño en órganos diana, siempre que no se planifique el embarazo.

La respuesta a la terapia farmacológica se debe evaluar a las 6-8 semanas desde el inicio del tratamiento o tras una intensificación del mismo con el objeto de valorar la respuesta alcanzada y posibles efectos secundarios del tratamiento y así continuar con la misma pauta, intensificar la misma si fuese necesario o plantear cambios en su caso. Como norma general, el seguimiento mediante analítica se realiza a los 6-12 meses, aunque este plazo es arbitrario. Como mínimo, se debe determinar el $c-L D L$ que constituye nuestro objetivo primario aunque se aconseja disponer de un perfil lipídico completo. También debe analizarse el colesterol no HDL o la ApoB (apolipoproteína B) y utilizarlos como dianas terapéuticas secundarias.

- Estatinas (lovastatina, pravastatina, fluvastatina, simvastatina, atorvastatina, rosuvastatina, pitavastatina): actúan disminuyendo la síntesis endógena de colesterol mediante inhibición competitiva de la enzima HMG-CoA reductasa o 3-hidroxi-3-metil-glutaril-CoA reductasa, enzima limitante en la biosíntesis del colesterol. El grado de reducción de c-LDL es de $30 \%$ a > $50 \%$, con amplia variabilidad interindividual. Se dispone de una tabla de equivalencias ${ }^{16}$. Reducen los TG un 10-20\%, siendo las más potentes las más eficaces. Elevan el c-HDL 1-10\%. No modifican la Lp(a) (lipoproteína (a)). Su eficacia en la reducción de eventos está demostrada tanto en Prevención Primaria como Secundaria. En el CTT (Cholesterol Treatment Trialists), el uso de estatinas versus control o un régimen de estatinas más intensivo versus menos intensivo ${ }^{17}$ por cada reducción de 33,8 mg/dl en
c-LDL se redujeron los eventos vasculares mayores (infarto de miocardio, muerte por enfermedad coronaria, cualquier accidente cerebrovascular o revascularización coronaria) en un $22 \%$, los eventos coronarios mayores en un $23 \%$, la muerte por enfermedad coronaria en un $20 \%$, el accidente cerebrovascular total en un $17 \%$ y la mortalidad total en un $10 \%$ durante cinco años.

Es evidente que las estatinas tienen beneficios notorios sobre la mortalidad y sobre los eventos cardiovasculares adversos en los pacientes con diabetes. Su efectividad es mayor con dosis altas que con dosis más bajas, al coste de un ligero aumento de los efectos secundarios. La reducción de riesgo relativo de eventos vasculares adversos con estatinas es similar en pacientes con diabetes que en pacientes no diabéticos, pero la reducción de riesgo absoluto es mayor en los pacientes con diabetes dada su mayor tasa de eventos.

- Ezetimiba: inhibe la absorción intestinal de colesterol dietético y biliar, sin afectar a la absorción de otros nutrientes liposolubles. Reduce el c-LDL un 15-22 \%. Eleva un $3 \%$ el c-HDL y disminuye un $8 \%$ Ios TG. Asociada a estatinas, reduce el c-LDL un 21$27 \%$. La administración con resinas puede producir una disminución adicional de c-LDL de un 10-20 \%. La administración con iPCSK9 también produce un efecto adicional. La eficacia de la ezetimiba en la prevención cardiovascular no se ha demostrado en monoterapia, pero sí en asociación con estatinas ${ }^{18}$, en pacientes con insuficiencia renal ${ }^{19}$ y en pacientes con síndrome coronario agudo ${ }^{20}$.

Dada la evidencia analizada, la asociación de ezetimiba y estatinas tiene un lugar en el tratamiento de aquellas personas con diabetes de alto riesgo que no toleren dosis altas de estatinas, o que no lleguen a las metas establecidas.

- Inhibidores de la PCSK9 (evolocumab, alirocumab): actúan bloqueando la PCSK9, con lo cual aumenta la expresión de los receptores hepatocitarios de $c-L D L$ en la superficie celular incrementando el aclaramiento de partículas de c-LDL. Reducen significativamente los niveles de c-LDL en promedio en un $60 \%$. En combinación con estatinas de alta intensidad, disminuyen el c-LDL en un 46-73 \% más que el placebo, y en un $30 \%$ más que la ezetimiba. También reducen los niveles de TG y aumentan los de c-HDL y ApoA1 (Apoproteína A1). A diferencia de las estatinas, estos fármacos sí reducen los 
niveles plasmáticos de Lp(a) aproximadamente un 30-40\%. Asociados a estatinas, en pacientes con cardiopatía isquémica estable en el FOURIER ${ }^{21}$ con evolocumab y tras síndrome coronario agudo en el ODYSSEY Outcomes ${ }^{22}$ con alirocumab, han demostrado reducción de eventos cardiovasculares.

Dada la evidencia analizada se debería considerar como terapia adyuvante en pacientes con diabetes con alto riesgo cardiovascular y que requieren reducción adicional de c-LDL luego de dosis máximas de estatinas, o que lo requieren y no toleran las altas dosis de estatinas.

- Resinas (colestiramina, colestipol, colesevelam): se unen a los ácidos biliares e impiden su absorción, aumentando en el hígado la expresión de receptores de c-LDL y captando más partículas de c-LDL del plasma. A la dosis diaria máxima se consigue una reducción en c-LDL de 18-25\%, no modifican el c-HDL, mientras que los TG pueden aumentar en algunos pacientes predispuestos. Solo la resincolestiramina ha demostrado que disminuye los eventos coronarios en los hombres en prevención primaria en la era pre-estatinas ${ }^{23}$.

- Fibratos (fenofibrato, gemfibrozilo, bezafibrato): son agonistas de los receptores PPAR-a (receptores activados por proliferadores peroxisómicos alfa). Reducen los TG en ayunas y posprandiales, así como el remanente de lipoproteínas ricas en TG. Logran una reducción del $50 \%$ del nivel de TG, $<20 \%$ del nivel de c-LDL e incrementan el nivel del c-HDL de $<20 \%$. Solo el gemfibrozilo ${ }^{24}$ en monoterapia ha demostrado reducción de eventos cardiovasculares, tanto en PP (Prevención Primaria) como en PS (Prevención Secundaria). El fenofibrato25, asociado a estatinas, podría reducir los eventos en pacientes con diabetes, siendo el único que posee indicación de su uso en combinación con estas.

- Ácidos grasos poliinsaturados de cadena larga omega-3 (AGPICL w-3) como el ácido eicosapentaenoico (EPA) y docosahexaenoico (DHA): inhiben la síntesis hepática de CVLDL. Además tienen otros efectos antitrombóticos, antiarrítmicos, protección endotelio, etc.). A dosis de 2-4 g/día pueden reducir los TG hasta un $45 \%$, aunque sus efectos sobre otras lipoproteínas son triviales. Una revisión Cochrane ${ }^{26}$ no informó de ningún efecto de los ácidos grasos omega-3 sobre la mortalidad total o por ECV, pero sí sobre los eventos coronarios. El uso de dosis $2 \mathrm{~g} / 12 \mathrm{~h}$ de EPA en comparación con placebo redujo el riesgo relativo en los principales eventos cardiovasculares en el estudio REDUCE-IT7 un $25 \%$ (en 8.179 pacientes, en PS, en tratamiento con estatinas, con c-LDL 40-100 mg/dl y varios $F R C V$, incluyendo $T G$ elevados persistentes entre 150 y $499 \mathrm{mg} / \mathrm{dl})$.

- Estrategias para controlar los triglicéridos del plasma. Aunque el riesgo de ECV aumenta cuando los TG en ayunas son > $150 \mathrm{mg} / \mathrm{dl}$, el uso de medicamentos para reducir los niveles de TG solo puede ser considerado en pacientes de alto o muy alto RCV (es decir, cuando los TG son > 200 mg/dl). Las hipertrigliceridemias responden muy bien a las medidas no farmacológicas, fibratos y ácidos grasos polinsaturados omega-327. Un nuevo modulador selectivo PPAR-a (pemafibrato) ha demostrado tener una marcada eficacia en la reducción de TG; con este fármaco se ha iniciado el estudio PROMINENTE $^{28}$ (10.000 pacientes con diabetes de alto riesgo con TG alto y niveles bajos de c-HDL).

\section{Perspectivas futuras}

El inclisiran ${ }^{29}$ es un ácido ribonucleico (ARN) de interferencia que bloquea la trascripción de la PCSK9, y que puede dosificarse cada seis meses (estudios en fase III). Por otra parte, el ácido bempedoico ${ }^{30}$ (estudios en fase III) es una molécula pequeña oral, primera en su clase que inhibe la síntesis de colesterol al bloquear la acción del citrato de ATP liasa, una enzima citosólica de la 3-hidroxi-3-metilglutarilcoenzima reductasa; hasta ahora se ha probado en pacientes con diabetes y en pacientes con o sin «intolerancia» a las estatinas. En monoterapia, el ácido bempedoico reduce los niveles de c-LDL en un $30 \%$ y en aproximadamente un $50 \%$ en combinación con ezetimiba. Puede que dispongamos de estas dos moléculas a lo largo de 2021. El volanesorsen ${ }^{31}$ disminuye los TG en un $70 \%$; la Agencia Europea de Medicamentos (EMA) lo ha autorizado como adyuvante a la dieta en adultos con familiares confirmados genéticamente con síndrome de quilomicronemia que están en alto riesgo de pancreatitis, en quienes la respuesta a la dieta y a la terapia reductora de TG ha sido inadecuada. Por otro lado, en pacientes con hipercolesterolemia refractaria, que tienen c-LDL elevado a pesar del tratamiento con terapias hipolipemiantes a las máximas dosis toleradas y un riesgo cardiovascular elevado, evinacumab ${ }^{32}$ subcutáneo e intravenoso, un anticuerpo monoclonal contra la angiopoyetina-like 3, redujo el nivel de colesterol LDL en más del $50 \%$ a la dosis máxima. 


\section{Bibliografía}

1. Estruch R, Ros E, Salas-Salvado J, Covas M-I, Corella D, Aros F, et al, for the PREDIMED Study Investigators. Primary Prevention of Cardiovascular Disease with a Mediterranean Diet Supplemented with Extra-Virgin Olive Oil or Nuts. N Engl J Med. 2018 Jun 21;378(25):e34. doi: 10.1056/NEJMoa1800389. Epub 2018 Jun 13

2. https://www.eas-society.org/page/dyslipidemia_ guide_2019.

3. Catapano A, Graham I, De Backer C, Wiklund O, Chapman MJ, Drexel H, et al. 2016 ESC/EAS Guidelines for the management of dyslipidaemias: the Task Force for the management of dyslipidaemias of the European Society of Cardiology (ESC) and the European Atherosclerosis Society(EAS). Eur Heart J. 2016;37(39):2999-3058.

4. Howard BV, Van Horn L, Hsia J, Manson JE, Stefanick ML, Wassertheil-Smoller S, et al. Low-fat dietary pattern and risk of cardiovascular disease: The Women's Health Initiative Randomized Controlled Dietary Modification Trial. JAMA. 2006;295:655-666. http://dx.doi.org/10.1001/jama.295.6.655

5. Pérez F, Pascual V, Félix J, Pérez P, DelgadoJ, Domenech $M$, et al. Documento de recomendaciones de la SEA 2018. El estilo de vida en la prevención cardiovascular. Clin Investig Arterioscler. 2018;30(6):280-310.

6. Burke FM. Red Yeast Rice for the Treatment of Dyslipidemia. Curr Atheroscler Rep. 2015;17(22):1-6.

7. https://www.acc.org/latest-in-cardiology/clinicaltrials/2018/11/08/22/48/reduce-it.

8. Wang $Y, X u$ D. Efectos del ejercicio aeróbico sobre lípidos y lipoproteínas. Lipids Health Dis 16, 132 (2017). https://doi.org/10.1186/s12944-017-0515-5.

9. Organización Mundial de la Salud (OMS) (2010). Recomendaciones mundiales sobre la actividad física para la salud. Ginebra, Suiza [consultado 1 Mar 2017, en: http://whqlibdoc.who.int/ publications/2010/9789243599977_spa.pdf

10. Boulé NG, Haddad E, Kenny GP, Wells GA, Sigal RJ. Effects of exercise on glycemic control and body mass in Type 2 Diabetes Mellitus: a metaanalysis of controlled clinical trials. JAMA. $2001 \mathrm{Sep}$ 12;286(10):1218-27. doi: 10.1001/jama.286.10.1218.

11. Colberg SR, Sigal RJ, Fernhall B, Regensteiner JG, Blissmer BJ, Rubin RR, et al. Exercise and type 2 diabetes: The American College of Sports Medicine and the American Diabetes Association: joint position statement. Diabetes Care. 2010 Dec;33(12):e147-e67. doi: 10.2337/dc10-9990.

12. Blomster II, Chow CK, Zoungas S, Woodward M, Patel A, Poulter NR, et al. The influence of physical activity on vascular complications and mortality in patients with type 2 diabetes mellitus. Diabetes Obes Metab. 2013 Nov;15(11):1008-12. doi: 10.1111/dom.12122.

13. Moe B, Eilertsen E, Nilsen TIL. The combined effect of leisure-time physical activity and diabetes on cardiovascular mortality. Diabetes Care. 2013 Mar;36(3):690-5. http://dx.doi.org/10.2337/dc112472.

14. Catapano AL, Graham I, De Backer G, Wiklund O, Chapman MJ, Drexel H, et al. 2016 ESC/EAS.Guidelines for the management of dyslipidaemias. Eur Heart J. 2016 Aug:ehw272. doi: 10.1093/eurheartj/ehw272.
15. Adiels $M$, Olofsson S-O, Taskinen M-R, Borén !. Overproduction of very low-density lipoproteins is the hallmark of the dyslipidemia in the metabolic syndrome. Arterioscler Thromb Vasc Biol. 2008. Jul;28(7):1225-36.doi: 10.1161/ATVBAHA.107.160192.

16. http://bit.ly/2VeJvnX.

17. Baigent C, Blackwell L, Emberson J, Holland LE, Reith C, Bhala N, et al.; Cholesterol Treatment Trialists Collaboration. Efficacy and safety of more intensive lowering of LDL cholesterol: a metaanalysis of data from 170,000 participants in 26 randomised trials. Lancet. 2010:376:1670-81.

18. Zhan S, Tang M, Liu F, Xia P, Shu M, Wu X. Ezetimibe for the prevention of cardiovascular disease and all-cause mortality events. Cochrane Database of Systematic Reviews 2018, Issue 11. Art. No.: CD012502. doi: 10.1002/14651858.CD012502.pub2. Accessed 01 January 2021.

19. Baigent C, Landray MJ, Reith C, Emberson J, Wheeler DC, Tomson C, et al; SHARP Investigators. The effects of lowering LDL colesterol with simvastatin plus ezetimibe in patients with chronic kidney disease (Study of Heart and Renal Protection): a randomized placebo-controlled trial. Lancet. 2011;377:2181-92.

20. Cannon CP, Blazing MA, Giugliano RP, McCagg A, White JA, Theroux P, et al; IMPROVE-IT Investigators. Ezetimibe added to statin therapy after acute coronary syndromes. N Engl J Med. 2015;372:2387-97.

21. Sabatine MS, Giugliono RP, Keech AC, Honarpour N, Wivlott SD, Murphy SA, et al. Evolocumab and clinical outcomes in patients with cardiovascular disease. N Eng J Med. 2017;376:1713-22.

22. Schwartz GG, Szarek SM, Bhatt DI, Bittner VA, Díaz $\mathrm{R}$, Edelberg JM, et al. Alirocumab and cardiovascular outcomes after acute coronary syndrome. N Eng J Med. 2018;379:2097-2107.

23. Third Report of the National Cholesterol Education Program (NCEP) Expert Panel on Detection, Evaluation and Treatment of High Blood Cholesterol in Adults (Adult Treatment Panel III). Circulation 2002; 106:3143-3421.

24. Bloomfield Rubins H, Robins SJ, Collins D y col. N Engl J Med. 1999,341:410-418.

25. Elam MB, Ginsberg HN, Lovato LC, et al. Association of Fenofibrate Therapy With Long-term Cardiovascular Risk in Statin-Treated Patients With Type 2 Diabetes. IAMA Cardiol. 2017;2(4):370-380. doi: 10.1001/ jamacardio.2016.4828.

26. Abdelhamid AS, Brown TJ, Brainard JS, Biswas P, Thorpe GC, Moore HI, Deane KHO, Summerbell CD, Worthington HV, Song F, Hooper L. Omega-3 fatty acids for the primary and secondary prevention of cardiovascular disease. Cochrane Database of Systematic Reviews 2020, Issue 3. Art. No.: CD003177. doi: 10.1002/14651858.CD003177.pub5.

27. https://www.semfyc.es/wp-content/ uploads/2020/09/Dislipemias-31-08-2020.pdf.

28. https://clinicaltrials.gov/ct2/show/NCT03071692.

29. https://www.nejm.org/doi/full/10.1056/NEJMoa 1912387.

30. https://jamanetwork.com/journals/jamacardiology/ fullarticle/2767760.

31. https://pubmed.ncbi.nlm.nih.gov/31390500/.

32. https://www.nejm.org/doi/full/10.1056/NEJMoa 2031049 